\title{
Исследование сегрегации сурьмы при эпитаксиальном росте на подложках Si c различной кристаллографической ориентацией
}

\author{
(С Д.В. Юрасов ${ }^{1,2}$, М.Н. Дроздов ${ }^{1}$, В.Б. Шмагин ${ }^{1}$, А.В. Новиков ${ }^{1,2}$ \\ ${ }^{1}$ Институт фризики микроструктур Российской академии наук, \\ 603087 Нижегородская обл., Россия \\ ${ }^{2}$ Нижегородский государственный университет им. Н.И. Лобачевского, \\ 603950 Нижний Новгород, Россия \\ E-mail: Inquisitor@ipmras.ru
}

(Получена 27 апреля 2017 г. Принята к печати 12 мая 2017 г.)

Исследована сегрегация $\mathrm{Sb}$ в слоях $\mathrm{Si}$, выращенных методом молекулярно-пучковой эпитаксии на подложках $\mathrm{Si}$ с кристаллографической ориентацией (111), (110), (115), и проведено сравнение полученных результатов для этих ориентаций с результатами для наиболее распространенной ориентации (001). Обнаружено, что качественно ход температурных зависимостей коэффициента сегрегации $\mathrm{Sb}(r)$ схож для всех исследованных ориентаций, в частности можно выделить два характерных температурных интервала, соответствующих режиму кинетических ограничений и термодинамически равновесному режиму сегрегации. Однако количественно данные для величины $r$ при исследованных ориентациях заметно отличаются от таковых для (001) при одинаковых температурах. Обнаруженные на зависимостях $r$ от температуры роста участки, где $r$ меняется на 5 порядков в узком интервале температур, позволили адаптировать метод селективного легирования, основанный на контролируемом использовании эффекта сегрегации, предложенный авторами ранее для структур на $\mathrm{Si}(001)$, к структурам, выращенным на подложках $\mathrm{Si}$ иных ориентаций. С помощью данного метода были изготовлены селективно-легированные структуры $\mathrm{Si}: \mathrm{Sb} / \mathrm{Si}(111)$, в которых изменение концентрации $\mathrm{Sb}$ на порядок происходит на масштабах в несколько нанометров.

DOI: $10.21883 /$ FTP.2017.12.45173.36

\section{1. Введение}

Одной из важных проблем при эпитаксиальном росте полупроводников является обеспечение наперед заданного распределения легирующих примесей. Хорошо известно, что процессы сегрегации, диффузии и десорбции примесей могут серьезнейшим образом искажать профиль распределения примесей, что при современных масштабах активных областей структур в десятки и единицы нанометров становится совершенно неприемлемым. Сегрегация при типичных условиях роста структур на кремниевых подложках оказывается процессом, как правило, наиболее сильно влияющим на перераспределение донорных примесей [1-3]. В подавляющем большинстве опубликованных работ по исследованиям сегрегации донорных примесей в кремнии рассматривались структуры, выращенные на подложках $\mathrm{Si}(001)$ [2-9], что связано с наибольшей технологической востребованностью подложек данной кристаллографической ориентации. Однако использование подложек с кристаллографической ориентацией, отличной от (001), может обеспечить некоторые преимущества, такие как увеличение подвижности носителей заряда [10,11], изменение зонной структуры и спиновых свойств структур, сформированных на высокоиндексных подложках [12]. $\mathrm{B}$ настоящее время пластины $\mathrm{Si}$ с ориентацией (111) успешно используются для выращивания на кремнии полупроводников типа $\mathrm{A}^{\mathrm{III}} \mathrm{N}[13,14]$. Кроме того, для некоторых приборных приложений ориентация $\mathrm{Si}(111)$ оказывается наиболее предпочтительной в силу макси- мально плотной упаковки атомов Si. B то же время, как было отмечено выше, сегрегация донорных примесей в Si-структурах, выращенных на подложках с ориентацией, отличающейся от $(001)$, изучена крайне мало $[1,15,16]$.

В настоящей работе приведены результаты исследований сегрегации сурьмы в кремниевых структурах, выращенных на подложках $\mathrm{Si}(111), \operatorname{Si}(110)$ и $\mathrm{Si}(115)$ методом молекулярно-пучковой эпитаксии, и проведено сравнение полученных результатов с данными для подложек $\mathrm{Si}(001)$. На основе полученных результатов методика селективного легирования Si сурьмой, разработанная авторами ранее для структур, выращенных на $\mathrm{Si}(001)$ [8], адаптирована для иных кристаллографических ориентаций подложек $\mathrm{Si}$. C ее помощью получены селективно легированные слои, в которых переходные области между легированным и нелегированным слоями имеют масштаб в несколько нанометров.

\section{2. Методика эксперимента}

Все исследованные структуры были выращены методом молекулярно-пучковой эпитаксии (МПЭ) на сверхвысоковакуумной установке Riber SIVA-21. Для осаждения кремния установка оснащена электронно-лучевым испарителем (ЭЛИ), а для осаждения $\mathrm{Sb}$ - стандартной эффузионной ячейкой Кнудсена. Включение квадрупольного масс-спектрометра в цепь обратной связи ЭЛИ позволяло поддерживать стабильность потока $\mathrm{Si}$ во время роста в пределах $\pm 5 \%$. Температура образцов измерялась с помощью специализированного инфракрас- 
ного пирометра IMPAC IS-12 и термопары, откалиброванной методом низкокогерентной тандемной интерферометрии $[17,18]$. Морфология поверхности образцов контролировалась in situ с помощью системы дифракции быстрых электронов (ДБЭ). В качестве подложек использовались пластины кремния с кристаллографическими ориентациями (001), (110), (111) и (115).

Для анализа распределения примесей по глубине использовалась вторично-ионная масс-спектрометрия (ВИМС, времяпролетный масс-спектрометр TOF.SIMS-5) и измерение вольт-фарадных характеристик $(\mathrm{B} \Phi \mathrm{X})$. Для увеличения разрешения по глубине в методе ВИМС использовался режим распыления ионами $\mathrm{Cs}^{+}$ c пониженной энергией (1 кэВ), а для увеличения чувствительности по $\mathrm{Sb}$ - регистрация не ионов $\mathrm{Sb}$, a кластерных ионов $\mathrm{SiSb}$. Количественная калибровка метода ВИМС осуществлялась путем анализа подложек c заранее известной концентрацией $\mathrm{Sb}$, а также за счет сравнения концентрации атомов $\mathrm{Sb}$ в однородно легированных тестовых образцах с концентрацией электронов, полученной из измерений эффекта Холла (в предположении полной электрической активации атомов $\mathrm{Sb}$ ). Для повышения воспроизводимости количественных калибровок ВИМС для различных структур при определении объемной концентрации $\mathrm{Sb}$ использовалась нормировка сигнала от кластерных ионов $\mathrm{SiSb}$ на сигнал от изотопа ${ }^{30} \mathrm{Si}$. Для исследования распределения $\mathrm{Sb}$ в структурах, в которых ее объемная концентрация была ниже чувствительности используемой аппаратуры ВИМС $\left(<5 \cdot 10^{16} \mathrm{~cm}^{-3}\right)$, использовалось измерение ВФХ на частотах 0.5-1МГц на установке QuadTech 7600 LCR-meter. Для формирования контактов использовалось термическое напыление $\mathrm{Al}$ (в планарной геометрии, размер контактов $\sim 500$ мкм).

\section{3. Результаты и их обсуждение}

Для количественного описания сегрегационных свойств $\mathrm{Sb}$ удобно использовать безразмерный коэффициент сегрегации $r$, определяемый как отношение поверхностной концентрации примеси к ее объемной концентрации, нормированное на толщину 1 монослоя [4]. В настоящей работе экспериментальное определение зависимости величины $r$ от температуры роста было выполнено за счет выращивания серии структур, в которых $\sim(0.7-1) \cdot 10^{14} \mathrm{~cm}^{-2}$ атомов $\mathrm{Sb}$ заращивались слоем $\mathrm{Si}$ при различных температурах, и исследования распределения атомов примеси по глубине в выращенных структурах с помощью ВИМС и измерений ВФХ. На рис. 1 показаны типичные профили распределения $\mathrm{Sb}$ по глубине, полученные с помощью ВИМС, для одной из исследованных температур $\left(450^{\circ} \mathrm{C}\right)$ в структурах, выращенных на подложках $\mathrm{Si}$ с различной кристаллографической ориентацией.

Из рис. 1 очевидны различия в выраженности эффекта сегрегации $\mathrm{Sb}$ в зависимости от кристаллографической

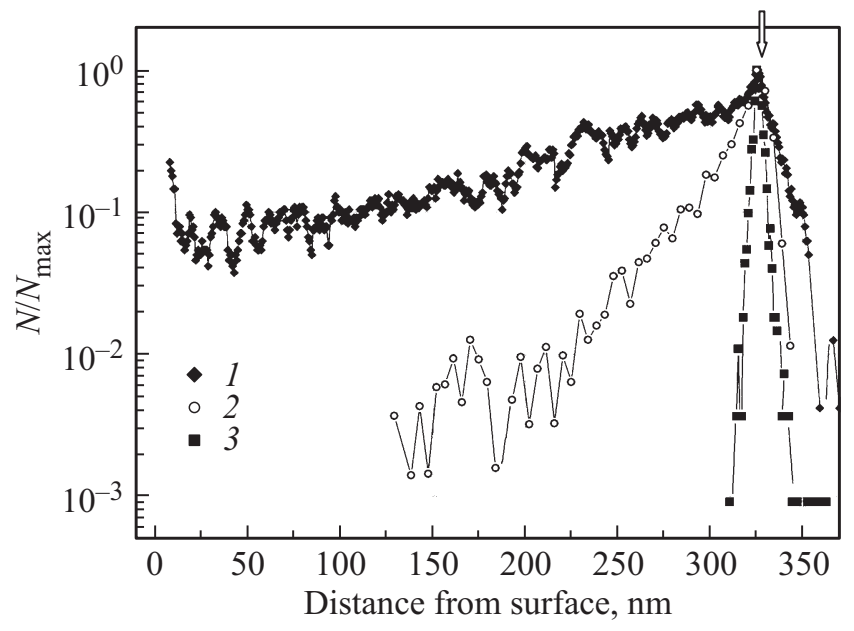

Рис. 1. Профили распределения концентрации $\mathrm{Sb} N$ по глубине в структурах, выращенных на подложках $\mathrm{Si}$ с разными кристаллографическими ориентациями: $1-(001), 2-(110)$, 3 - (111). Стрелкой отмечено место осаждения $\delta$-слоя $\mathrm{Sb}$. Каждый из профилей для наглядности нормирован на свой максимум $N_{\max }$.

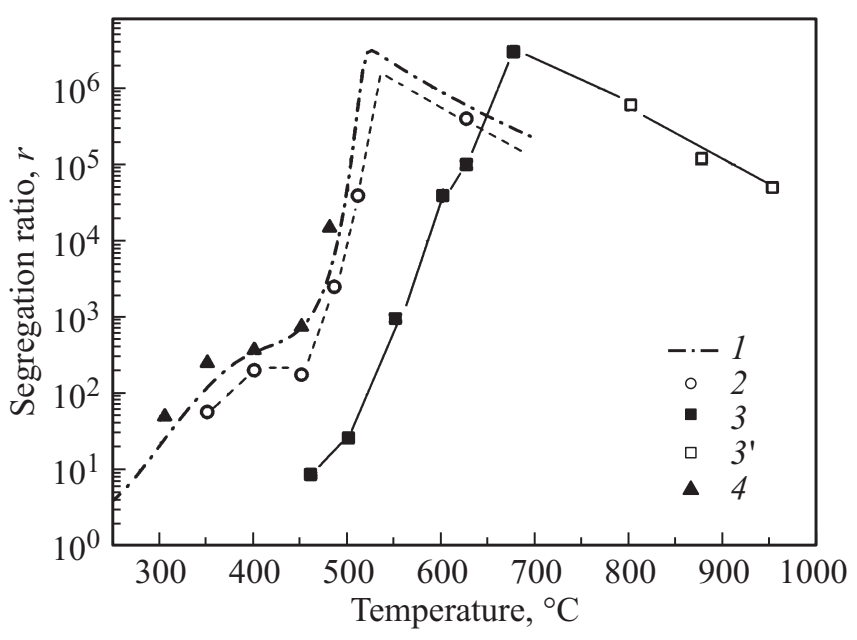

Рис. 2. Температурные зависимости коэффициента сегрегации $\mathrm{Sb}$ для различных ориентаций подложки $\mathrm{Si}: 1-(001)$ (из [6]), 2 - (110), 3 - (111), $3^{\prime}-(111)$ (из [19]), 4 - (115). 2, 3, 4 - данные настоящей работы.

ориентации подложки $\mathrm{Si}$. Значение $r$ из профилей концентрации $\mathrm{Sb}$ в выращенных структурах, полученных с помощью ВИМС, определялось по методике, предложенной в [4]. Оценка значения $r$ из измерений ВФХ была сделана путем вычисления нормированного отношения изначально высаженного количества $\mathrm{Sb}$ к величине средней объемной концентрации. Измерения ВФХ проводились для структур, в которых $r$ было велико $\left(>10^{5}\right)$, поэтому спад объемной концентрации с толщиной происходил крайне медленно. В связи с этим объемная концентрация $\mathrm{Sb}$ слабо менялась по толщине слоя (вдали от границ), и при вычислениях было взято 
ее среднее значение. Результаты серии аналогичных экспериментов, проведенных при различных температурах, а также литературные данные из работ $[6,19]$ представлены на рис. 2.

Можно отметить, что вид температурных зависимостей $r(T)$ для различных ориентаций подложки $\mathrm{Si}$ оказывается схожим. Наблюдаются два характерных температурных режима сегрегации, а именно режим кинетических ограничений $(r$ растет с ростом $T)$ и термодинамически равновесный режим $(r$ убывает с ростом $T$ ), а также резкий рост $r$ с увеличением $T$ вблизи перехода от кинетически-ограниченного к равновесному режиму. Однако количественно полученные зависимости $r(T)$ для различных кристаллографических ориентаций имеют существенные различия. Например, температура перехода между режимами сегрегации смещена для $\mathrm{Si}(111)$ примерно на $150-170^{\circ} \mathrm{C}$ в сторону более высоких температур по сравнению с $\mathrm{Si}(001)$.

Детальное рассмотрение зависимостей $r(T)$ для подложек $\mathrm{Si}$ с различной кристаллографической ориентацией удобно начать с ориентации (115), зависимость $r(T)$ для которой оказалась наиболее близка к наблюдаемой для (001). Исследования, проведенные в работе [20] методом сканирующей туннельной микроскопии, выявили, что поверхность $\mathrm{Si}(115)$ имеет в общем схожую с (001) структуру, а именно „террасы + ступени“. Однако в силу большого угла отклонения поверхности (115) от (001), который составляет $\sim 15.8^{\circ}$, поверхность $(115)$ имеет более сложную организацию, а ширина террас на ней составляет всего $\langle 2$ димера $\rangle+\langle 1$ тетрамер $\rangle$ (тетрамер - димер, связанный с 2 атомами края ступени) [20]. В некотором достаточно грубом приближении такую поверхность можно рассматривать как поверхность типа „(001) с короткими террасами“. Согласно модели [6], в кинетически-ограниченном режиме сегрегации, когда основным механизмом является сегрегация на моноатомных ступенях, величина $r$ пропорциональна количеству мест, где атом примеси может перейти с нижней террасы на верхнюю. Иными словами, величина $r$ пропорциональна „плотности“ моноатомных ступеней, т.е. обратно пропорциональна длине моноатомных террас. Рассматривая поверхность (115) как поверхность „(001) с короткой террасой“, получаем, что при прочих равных условиях для поверхности с более короткими террасами $r$ в режиме кинетически-ограниченной сегрегации должен быть выше, что и наблюдалось в эксперименте (рис. 2). С другой стороны, не очевидно, что потенциальный барьер для процесса „вскарабкивания“ атома примеси на ступени будет одинаков для моно- и двухатомных ступеней. Более полное теоретическое описание процесса сегрегации в структурах, выращенных на подложках $\mathrm{Si}(115)$, требует дальнейших исследований, в частности получения экспериментальных данных для равновесного и переходного режимов сегрегации, что позволило бы оценить величину такого барьера в модели [6].

Исследования сегрегации Sb в структурах, выращенных на подложках $\operatorname{Si}(110)$ и $\operatorname{Si}(111)$, показали, что, несмотря на качественную схожесть кривых $r(T)$ для этих ориентаций, наблюдаются значительные количественные отличия от данных для $\mathrm{Si}(001)$ (в большей степени это касается ориентации (111)). Однако прямое применение теоретических моделей сегрегации примесей, изначально разработанных для $\mathrm{Si}(001)$, для ориентаций (110) и (111) лишь с некоторой численной корректировкой представляется затруднительным. Это связано со значительными различиями в структуре поверхностей кремния (001), (110) и (111), разной реконструкцией, разной конфигурацией оборванных связей и т. п. [20,21]. Поскольку эффект сегрегации является поверхностным эффектом, изменение микроструктуры поверхности может сильно сказываться на его величине. В связи с этим разработка теоретических моделей, учитывающих микроструктуру поверхности для различных граней кристалла $\mathrm{Si}$, требует дальнейших исследований.

Необходимо отметить, что общей чертой для полученных в настоящей работе зависимостей $r(T)$ для различных кристаллографических ориентаций подложек $\mathrm{Si}$ является наличие участка, разница в значениях величины $r$ в котором может достигать 5 порядков величины в достаточно узком интервале температур роста (см. рис. 2). Столь резкая зависимость $r(T)$ позволила адаптировать к кремниевым структурам, выращенным на подложках $\mathrm{Si}(110)$ и $\mathrm{Si}(111)$, метод селективного легирования, основанный на контролируемом использовании эффекта сегрегации и предложенный авторами ранее для структур, выращенных на $\mathrm{Si}(001)$. Подробное описание метода приведено в работе [8], а его суть заключается в использовании для роста легированных слоев достаточно низких температур, при которых значение $r$ невелико, а для роста нелегированных - температур, где $r$ близко к максимуму. Однако выбор температуры роста легированных слоев обусловлен не только тем, какую объемную концентрацию примеси необходимо получить, но и снижением кристаллического качества эпитаксиальных структур при низких температурах роста [22]. Известно, что атомы сурьмы, накопленные на поверхности роста вследствие сегрегации, оказывают существенное влияние на длину диффузии адатомов и кинетику ростовых процессов при эпитаксиальном росте структур на основе $\mathrm{Si}$ и Ge (см., например, [23]). При этом влияние $\mathrm{Sb}$ на ростовые процессы проявляется даже при ее малых поверхностных концентрациях (0.01 монослоя) [24], что заметно меньше тех концентраций, которые используются в предлагаемом методе селективного легирования [8].

В связи с этим для выявления влияния $\mathrm{Sb}$ на процессы роста кремния была проведена серия экспериментов, в которой при постоянном потоке $\mathrm{Sb}$ $\left(\sim 1.5 \cdot 10^{11} \mathrm{~cm}^{-2} \cdot \mathrm{c}^{-1}\right)$ при различных температурах на подложки $\mathrm{Si}(111)$ осаждались слои кремния. Контроль за изменением морфологии поверхности формируемых структур осуществлялся с помощью дифракции быстрых электронов (ДБЭ). Для сравнения также выращивалась аналогичная серия структур, но при отсутствии 

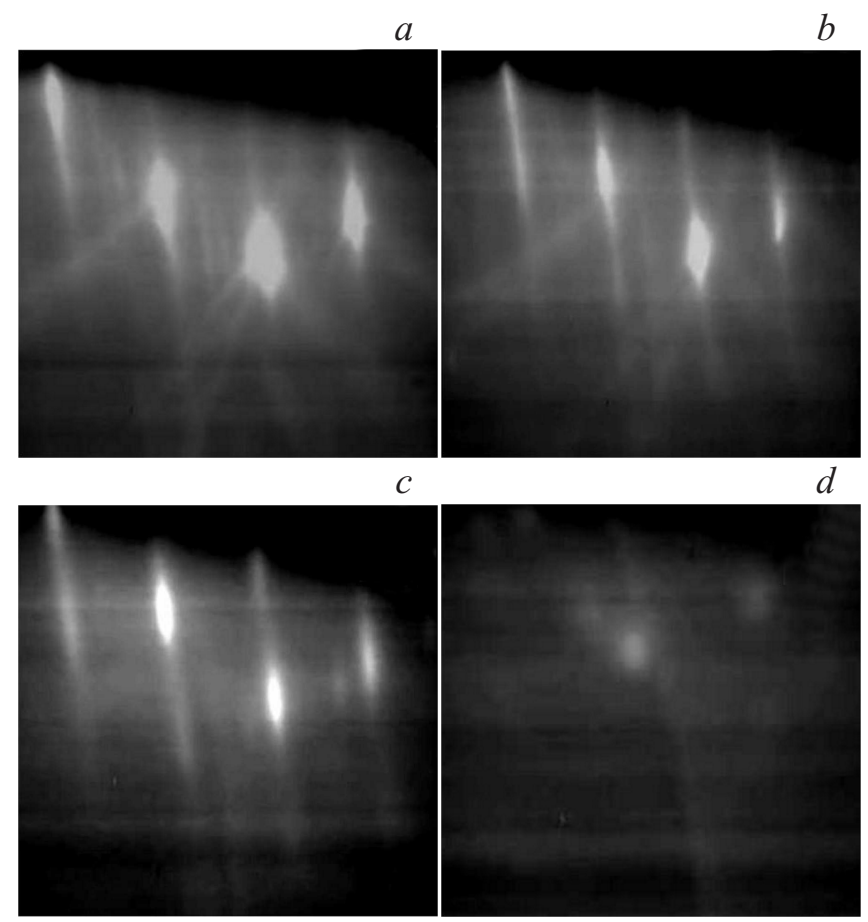

Рис. 3. Картины ДБЭ после роста слоев $\mathrm{Si}: \mathrm{Sb}$ толщиной 50 нм при: $500(a), 450(b), 400(c)$ и $350^{\circ} \mathrm{C}(d)$.

потока Sb. В обеих сериях экспериментов скорость осаждения Si была фиксирована на уровне $0.1 \mathrm{Hm} / \mathrm{c}$. Выбор ориентации (111) для данной серии экспериментов обусловлен практически полным кинетическим подавлением сегрегации Sb в структурах, выращенных на $\mathrm{Si}$ этой ориентации, при более высоких температурах по сравнению с другими ориентациями подложек $\mathrm{Si}$ $\left(T \approx 450^{\circ} \mathrm{C}\right.$, см. рис. 2$)$. Чтобы исключить влияние поверхностных загрязнений, а также для начальной проверки качества поверхности на подложку после ее термической очистки осаждался при температуре $740^{\circ} \mathrm{C}$ буферный слой $\mathrm{Si}$ толщиной 100 нм. Во всех случаях после роста такого буфера наблюдалась картина ДБЭ, соответствующая реконструкции $(7 \times 7)$, что свидетельствовало о формировании атомарно-чистой поверхности $\mathrm{Si}(111)$ [25]. Результаты изменения картины ДБЭ при варьировании температуры роста слоев $\mathrm{Si}$ в потоке $\mathrm{Sb}$ представлены на рис. 3.

Исходя из полученных картин ДБЭ можно указать, что переход от так называемой „полосковой“ картины, характерной для двумерного режима роста, к „точечной“, характерной для развития шероховатости поверхности, происходит при температурах $<500^{\circ} \mathrm{C}$, а при снижении температуры до $350^{\circ} \mathrm{C}$ происходит аморфизация растущей пленки. В контрольной серии образцов, выращенных без потока $\mathrm{Sb}$, аналогичный переход от „полосковой“ при температурах $\sim(400-450)^{\circ} \mathrm{C}$, что согласуется с данными, опубликованными в работе [15]. Согласно [23],
$\mathrm{Sb}$ как сурфактант значительно снижает диффузионную длину адатомов $\mathrm{Si}$, что для фиксированной температуры роста приводит к значительному увеличению плотности двумерных (2D) островков, которые, впоследствии срастаясь, формируют сплошной слой. Авторами [23] на основе исследований роста относительно тонких слоев $\mathrm{Si}$ (от субмонослойных покрытий и до 5 монослоев) на поверхности $\mathrm{Si}(111)$ с предосажденным 1 монослоем $\mathrm{Sb}$ был сделан вывод о том, что снижение диффузионной длины и многократное возрастание плотности 2D островков не препятствует послойному росту. Однако выполненные в настоящей работе исследования показывают, что для более толстых слоев $\mathrm{Si}: \mathrm{Sb}$ снижение диффузионной длины адатомов (особенно при низких температурах роста) будет приводить к увеличению поверхностной плотности дефектов/отклонений от идеальной реконструкции на поверхности $\mathrm{Si}$ (возникших от поверхностных загрязнений, локальных флуктуаций потока атомов, изломов ступеней и т. п.) и развитию ее шероховатости.

Таким образом, при формировании слоев $\mathrm{Si}: \mathrm{Sb} / \mathrm{Si}(111)$, толщина которых больше нескольких десятков нм, нежелательно использовать температуры роста ниже $500^{\circ} \mathrm{C}$. В методе селективного легирования, разработанном авторами ранее для структур, выращенных на $\mathrm{Si}(001)$ [8], и адаптированном в настоящей работе к структурам на $\mathrm{Si}(111)$, использовался интервал температур $520-700^{\circ} \mathrm{C}$. В данном интервале температур роста, с одной стороны, изменение величины $r$ составляет более 4 порядков величины (рис. 2), что позволяет создавать большие градиенты объемной концентрации примеси, а с другой стороны, не происходит заметной деградации кристаллического качества формируемых структур и развития высокой шероховатости поверхности даже при росте относительно толстых (сотни нм) легированных слоев. В частности, при формировании слоя $\mathrm{Si}: \mathrm{Sb}$ толщиной 100 нм с объемной концентрацией $\mathrm{Sb}$ $\sim 10^{19} \mathrm{~cm}^{-3}$ на подложке $\mathrm{Si}(111)$ использовалась температура $T=520^{\circ} \mathrm{C}$, когда значение $r$ невелико, а для формирования резкой границы профиля концентрации примеси использовалась остановка роста, во время которой температура подложки повышалась до $T=700^{\circ} \mathrm{C}$, при которой $r$ для ориентации (111) близко к максимуму (см. рис. 2). Рост нелегированных слоев $\mathrm{Si} / \mathrm{Si}(111)$ проходил при этой температуре. При использовании данного подхода были получены селективно-легированные структуры $\mathrm{Si}: \mathrm{Sb} / \mathrm{Si}(111)$, в которых ширина переходной области между легированным и нелегированным слоями, согласно измерениям ВИМС, составляла 1.5-3 нм (оценка проводилась по изменению объемной концентрации $\mathrm{Sb}$ на порядок), что сопоставимо со значениями, полученными авторами ранее для структур $\mathrm{Si}: \mathrm{Sb} / \mathrm{Si}(001)$ [8]. Последнее свидетельствует об успешности адаптации методики селективного легирования $\mathrm{Si}$ для различных кристаллографических ориентаций подложки $\mathrm{Si}$ при соответствующей корректировке температур роста. 


\section{4. Заключение}

В работе исследован процесс сегрегации $\mathrm{Sb}$ в кремнии, выращенном методом МПЭ на подложках $\mathrm{Si} \mathrm{c}$ различной кристаллографической ориентацией. Обнаружено, что качественно вид температурной зависимости коэффициента сегрегации оказывается схожим для различных ориентаций, а именно наблюдаются два характерных режима сегрегации - равновесный и режим кинетических ограничений. Однако количественно величина эффекта сегрегации при фиксированной температуре заметно отличается для разных ориентаций подложки $\mathrm{Si}$. Полученные температурные зависимости коэффициента сегрегации Sb позволили адаптировать метод селективного легирования, ранее предложенный авторами для $\mathrm{Si}(001)$, для других ориентаций подложек $\mathrm{Si} . \mathrm{C}$ помощью данного метода на подложках $\mathrm{Si}(111)$ были изготовлены селективно-легированные $\mathrm{Si}: \mathrm{Sb}$-структуры, в которых изменение концентрации $\mathrm{Sb}$ на порядок происходит на масштабах в несколько нанометров.

Работа выполнена при финансовой поддержке РФФИ (проект № 16-02-00686) с использованием оборудования ЦКП „Физика и технология микро- и наноструктур“ ИФМ РАН.

\section{Список литературы}

[1] R.A. Metzger, F.G. Allen. J. Appl. Phys., 55, 931 (1984).

[2] H. Jorke. Surf. Sci., 193, 569 (1988).

[3] H.J. Gossmann, E.F. Schubert. Crit. Rev. Sol. St. Mater. Sci., 18, 1 (1993).

[4] K.D. Hobart, D.J. Godbey, M.E. Twigg, M. Fatemi, P.E. Thompson, D.S. Simons. Surf. Sci., 334, 29 (1995).

[5] J.F. Nutzel, G. Abstreiter. Phys. Rev. B, 53, 13551 (1996).

[6] C.B. Arnold, M.J. Aziz. Phys. Rev. B, 72, 195419 (2005).

[7] I. Berberzier, J.P. Ayoub, A. Ronda, M. Oehme, K. Lyutovich, E. Kasper, M. Di Marino, G. Bisognin, E. Napolitani, M. Berti. J. Appl. Phys., 107, 034309 (2010).

[8] D.V. Yurasov, M.N. Drozdov, A.V. Murel, M.V. Shaleev, N.D. Zakharov, A.V. Novikov. J. Appl. Phys., 109, 113533 (2011).

[9] D.V. Yurasov, M.N. Drozdov, N.D. Zakharov, A.V. Novikov. J. Cryst. Growth, 396, 66 (2014).

[10] T. Low, M.F. Li, C. Shen, Y.-C. Yeo, Y.T. Hou, C. Zhu, A. Chin, D.L. Kwong. Appl. Phys. Lett., 85, 2402 (2004).

[11] A. Majumdar, C. Ouyang, S.J. Koester, W. Haensch. IEEE Trans. Electron. Dev., 57, 9 (2010).

[12] E. Gatti, F. Isa, D. Chrastina, E. Muller Gubler, F. Pezzoli, E. Grilli, G. Isella. J. Appl. Phys., 116, 043518 (2014).

[13] J.D. Brown, R. Borges, P.E. Vescan, S. Singhal, R. Therrien. Solid State Electron., 46, 1535 (2002).

[14] H.P.D. Schenk, E. Frayssinet, A. Bavard, D. Rondi, Y. Cordier, M. Kennard. J. Cryst. Growth, 314, 85 (2011).

[15] K. Nakagawa, M. Miyao, Y. Shiraki. Thin Sol. Films, 183, 315 (1989).

[16] M. Ladeveze, F. Bassani, F. Arnaud d'Avitaya, G. Treglia. C. Dubois, R. Stuck. Phys. Rev. B, 56, 7615 (1997).
[17] П.В. Волков, А.В. Горюнов, А.Ю. Лукьянов, А.Д. Тертышник, А.В. Новиков, Д.В. Юрасов, Н.А. Байдакова, Н.Н. Михайлов, В.Г. Ремесник, В.Д. Кузьмин. ФТП, 46, 1505 (2012).

[18] D.V. Yurasov, A.Yu. Luk'yanov, P.V. Volkov, A.V. Goryunov, A.D. Tertyshnik, M.N. Drozdov, A.V. Novikov. J. Cryst. Growth, 413, 42 (2015).

[19] S.A. Barnett, G.E. Greene. Surf. Sci., 151, 67 (1985).

[20] A.A. Baski, S.C. Erwin, L.J. Whitman. Surf. Sci., 392, 69 (1997).

[21] G.-H. Lu, M. Huang, M. Cuma, F. Liu. Surf. Sci., 588, 61 (2005).

[22] D.J. Eaglesham, H.-J. Gossmann, M. Cerullo. Phys. Rev. Lett., 65, 1227 (1990).

[23] B. Voigtlander, A. Zinner, T. Weber, H.P. Bonzel. Phys. Rev. B, 51, 7583 (1995).

[24] G.G. Jernigan, P.E. Thompson. Thin Sol. Films, 380, 1147 (2000).

[25] K. Takayanagi, Y. Tanishiro, S. Takahashi, M. Takahashi. Surf. Sci., 164, 367 (1985).

Редактор Л.В. Шаронова

\section{Antimony segregation in Si layers grown by MBE on Si wafers with different crystallographic orientations}

\author{
D.V. Yurasov ${ }^{1,2}$, M.N. Drozdov ${ }^{1}$, V.B. Schmagin ${ }^{1}$, \\ A.V. Novikov ${ }^{1,2}$ \\ ${ }^{1}$ Institute for Physics of Microstructures, \\ Russian Academy of Sciences, \\ 603087 Nizhny Novgorod region, Russia \\ ${ }^{2}$ Lobachevsky State University of Nizhny Novgorod, \\ 603950 Nizhny Novgorod, Russia
}

\begin{abstract}
Antimony segregation in Si layers grown by molecular beam epitaxy on Si wafers with (111), (110) and (115) crystallographic orientations was studied and the results were compared with the data for most widely used (001) orientation. The qualitative similarity of the temperature dependences of the segregation ratio $(r)$ was obtained for all investigated wafer orientations. In particular, two typical temperature intervals which correspond to the kinetically-limited and equilibrium segregation regimes were observed. But quantitatively the $r$ values for different wafer orientations differ significantly from the data for the $\mathrm{Si}(001)$ case at the same temperature. For all orientations the small temperature intervals within which the $r$ values varied by nearly 5 orders of magnitude were revealed. The latter allowed to adopt the technique of selective doping which was previously developed by the authors for $\mathrm{Si}(001)$ case to the structures grown on non-(001) Si wafers. Using this technique the selectively doped $\mathrm{Si}: \mathrm{Sb} / \mathrm{Si}(111)$ structures were fabricated in which the doping profile abruptness was of the order of a few nanometers.
\end{abstract}

